\title{
An Active Broadcast System for Safety-Oriented Facilities in Decentralized TDMA Assisted VANETs
}

\author{
A. Tamilarasi ${ }^{1 *}$, D. Sivabalaselvamani ${ }^{1}$, L. Rahunathan ${ }^{1}$, N. Adhithyaa ${ }^{1}$ \\ 1 Kongu Engineering College, Perundurai, Erode, 638060, India \\ * Corresponding author's e-mail: drtamil@kongu.ac.in
}

\begin{abstract}
In data link layer for vehicular Ad hoc Network, a twelve digit access control mechanism is available. That is Medium Access Control (MAC) protocol should be able to accommodate a wide variety of safety oriented real time applications in vehicular ad-hoc networks (VANETs). The most important aspect of those applications is the transmission safety messages in which we call it as Basic Safety Messages (BSMs) through V2V and V2I systems. When traffic thickness is heavy, a scheduled transfer of BSMs places a large load on the VANETs' stack, compromising network compatibility and performance. This article addresses a Decentralized TDMA-based framework for regulating transmission boundaries in VANETs to increase the potential of BSM transmissions. The transmitting effects are often low, but they do not reduce the consciousness of the nodes in close proximity. Consolidating packet collisions are often observed and cutoff at that time by a concerted effort among the nodes. Our procedures will provide a profitable and efficient BSM broadcasting, according to the simulation results.
\end{abstract}

Keywords: VANETs, broadcasting services, TDMA-MAC, performance evaluation.

\section{INTRODUCTION}

In VANETs the smart transportation systems that is aiming to increase the driver safety related applications is having nice proficiency in near future. Any vehicle is expected to send Basic Security Messages (BSMs) containing its status data (e.g., location, distance and bearing) will be sent to all the vehicles in a particular RSU on a regular basis such that all available vehicles in its range will get the current data in a scheduled time. This data is needed for the proposed driver well-being applications for VANETs. These systems need exceptionally high-quality status data from neighbouring nodes, which means that BSMs must be transmitted as often as possible $[4,10]$. A wide range of monitoring is also necessary, which necessitates a wide transmission range. Regardless, when traffic thickness is high, any node that is responsible to broadcast the defined safety messages in a single attempt by the help of wide message sending scope will cause heavy correspondence loads. When communicative loads are heavy, collisions in communicative standards of IEEE 802.11p have a great impact by which the delay time period is unbounded; even TDMA-based conventions [2, 7] have huge of accident rates when desired traffic thickness seems to be overly high. With the rise in packet crashes, heavy communicative stacks have a negative effect on vehicle consciousness consistency. Then there are incident-driven health signals that are transmitted in the event of a disaster, which are also important for vehicular stability, as the flagging weight must be kept to a minimum.

To minimize the detrimental effects of highthickness situations, the technique of regulating communicative range by adjusting the transmitting force is frequently used; however, a dynamic message sending frequency plot is commonly benefited in IEEE 802.11p [5]. When traffic thickness is high, much of the planned works adjust the transmitting capacity based on the surrounding vehicle thickness; in this manner, the transmission scope of vehicles is reduced to form a solid transmission. When the thickness is high, though, a lower transmission area collects just a 
few smaller consciousness ranges, implying that the packets must be obtained from usable cars. In, a randomization figure to determine an accurate transmitting power for VANET health-related applications, which offers much better consciousness efficiency at short distances while allowing for a more broad consciousness spread. Nonetheless, it is attempting to eradicate the issue of secret hubs, because it is impossible for hubs to assist one another when parcels are produced and communicated randomly.

The transmission power shift mechanism is commonly used in TDMA-based conventions to reduce power consumption and extend battery life [3]. Despite this, the concentration of few analysts of this approach increases the performance of sending safety messages to all vehicles in RSU's range. There are several conventions that involves TDMA, mentioned in, will have a cycle of timing slot shift strategy to assist successful transmission administrations. Nonetheless, a long length transmissions ends up with low acquiring rate, that can further degrade consciousness efficiency. In VANETs, vehicles may specifically assist one another. Under TDMA-based conventions, the transmission situation [9] and thus the power to be used are frequently facilitated among vehicles. We propose a completely new strategy that allows vehicles to use different transmission forces to fulfil different Observational locales, resulting in higher consciousness consistency and a wider consciousness spectrum. Around the same time, the cars will agreeably pre-identify and eliminate collision-related crashes; additionally, much stronger and successful broadcasting is achieved.

\section{BROADCASTING CONSIDERING DIFFERENT OBSERVATIONAL REGIONS}

Vehicles recognized data on different vehicles based on BSMs broadcasted by surrounding vehicles on a daily basis. If it will be invigorated even more often, the experience of an accompanying vehicle is becoming more specific. Since the distance between the sender and the authority is so small, the data accuracy should be higher.

\section{Various observational areas}

For the goal of a vehicle being able to detect dangerous factors in surrounding vehicles in real time, a large consciousness spectrum is expected. When it comes to cars that are at a distance, the response time is sufficient if an incident arises, and hence the treatment for these vehicles is often neglected. In either case, for vehicles approaching in close proximity, precise status information is required. Here, two distinct observational regions are depicted:

- Region of High Observational Performance (RHOP): RHOP, which fuses the area that is nearer for the node, necessitates extreme caution. Here, there will be an accurate information about the vehicle in RHOP.

- Region of Low Observational Performance (RLOP): In RLOP, a wider variety is preferable. The transmitting range is the most critical RLOP limit on the verge of collapsing. Furthermore, precise and up-to-date vehicle information isn't needed as much as it once required.

A massive transmission power $\mathrm{Pow}_{1}$ is needed to achieve RLOP. Meanwhile, since the RHOP is so small, small transmitting power Pow0 is often used to fulfil it.

\section{Switching transmission powers}

We get a completely new strategy using swapping of transmission powers Pow $_{0}$ and Pow $_{1}$ to accomplish a more wide range of better consciousness efficiency for closer vehicles [8]. As power $\mathrm{P}_{1}$ is used, area RHOP is always fulfilled, and RLOP is always fulfilled. Figure 1c illustrates the thought process using a model of the vehicles $\mathrm{X}$ and $\mathrm{Y}$. Vehicles $\mathrm{X}$ and $\mathrm{Y}$ use separate transmitting power orders to coordinate. The node points will accept data and use information about their neighbors since the sending situation was rendering by allocating MAC in a time slotted manner [6]. Our designed VANET system makes sending time and power are often formed together to understand higher capability. About the fact that the gap between node points $\mathrm{X}$ and $\mathrm{Y}$ is less than $2 \mathrm{Reg}_{1}$, they would happily use similar timeslots as long as their distance is greater than $\operatorname{Reg}_{1}+\operatorname{Reg}_{0}$, where $\operatorname{Reg}_{1}$ and $\operatorname{Reg}_{0}$ refer to the transmitting ranges when sent with powers Pow $_{1}$ and Pow respectively. In such a case, node points $\mathrm{X}$ and $\mathrm{Y}$ had to use separate time distributions in regular TDMA-based MAC displays, implying that the network would accommodate more node points by embracing our structure.

When transmitting capacity Pow $_{0}$ is used, the transmission range is reduced, and transmission results are also reduced. The signal weight should 

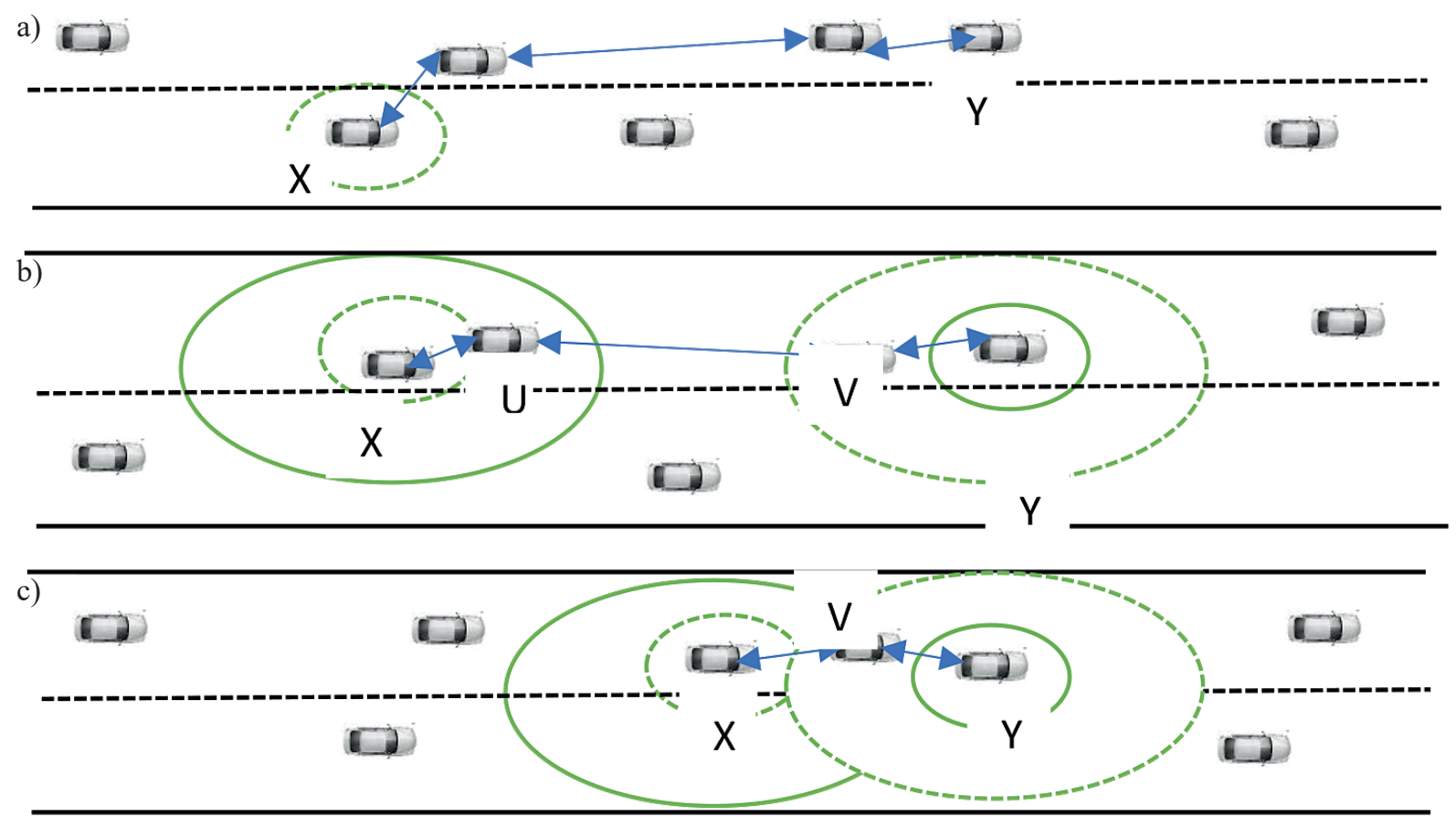

Fig. 1. a) Huge transmission power at both regions; b) Huge transmission power at Reg ; c) Less transmission power at both the regions

be minimized so that cars farther away will not get the signal. Certain signal combining problems are often pre-perceived and performed at that stage, reducing the message collision rate even further. Since node point $\mathrm{V}$ knows that node points $\mathrm{X}$ and $\mathrm{Y}$ use comparative timeslots, the running state of $\mathrm{X}$ and $\mathrm{Y}$ will also forecast a joining collapse, as seen in Figure 1c. If a car crash is likely, $\mathrm{V}$ will send out a slot swapping alert, instructing one of the possible node points to retake another slot before the accident happens.

In a switching mode, each node point uses transmission powers Pow $_{0}$ and Pow $_{1}$. The use of the power is denoted by the notation Pow $_{\mathrm{x}}$. Pow $_{\mathrm{x}}=$ 1 if node point $\mathrm{x}$ uses power Pow $_{1}$ for even-numbered time frames and power Pow $_{0}$ for odd-numbered time frames, otherwise Pow $_{\mathrm{x}}=0$. Pow $_{\mathrm{x}}$ is not the same as Pow ${ }_{y}$, as seen in Figure 1c. At the same time, node point node $\mathrm{X}$ is unable to switch the transmitting power demand; as a result, collisions will occur. We depicted a vector $\mathrm{D}_{\mathrm{x}}$ to see whether the transmitting power is often converted in $\mathrm{X} . \mathrm{D}_{\mathrm{x}}$ $=1$, when failure results followed by the vehicle point $\mathrm{x}$ changes its bands; else, $\mathrm{D}_{\mathrm{x}}=0$.

\section{Network cooperation}

Inside the TDMA-based MAC, every available node in the network should secure or acquire the space in the allotted time slots in order to send the packets to other vehicles in the range; it must also continue the previous obtained number of slots (opening) before a collision in sending up of the packets. When broadcasting an information, we also attach the basic information about the vehicles (e.g., ID, slot, Pow ${ }_{x}, D_{x}$ ) from all of the one-hop neighbours; Testing messages received by one hop neighbours will include details about a vehicle's two hop neighbours. During the reacquisition of a community, a car can choose a free slot that has not been used previously. If no such latent opening occurs at an event, then it selects a vehicle within $\operatorname{Reg}_{0}+\operatorname{Reg}_{1}$ distance. Variations of transmitting power should be used when getting to the channel during the slots, and the preferred opening should be used mainly by one neighbour node point.

In our proposed system, there are two types of packet collisions: Reconcilable Joining (RJ is vehicles competing for a single slot and use it using the Pow inside the scope of $\operatorname{Reg}_{0}+\operatorname{Reg}_{1}$ and $2 \mathrm{Reg}_{1}$, and Irreconcilable Joining (IRJ), is vehicles competing for the required space using separate $\mathrm{P}_{\mathrm{x}}$ under area not greater to $\operatorname{Reg}_{0}+\operatorname{Reg}_{1}$. The two kinds of packet collisions are often discarded with the assistance of neighbour node points.

A RJ happens as node points $\mathrm{X}$ and $\mathrm{Y}$ shift closer to each other, as seen in Figure 1a, and the distance between them falls below $2 \mathrm{Reg}_{1}$. Since of node point is aware of its two-hop neighbours 
initial use of information, node points $\mathrm{U}$ and $\mathrm{V}$ will anticipate the possible RJ [11]. Similarly, nodes $\mathrm{X}$ and $\mathrm{Y}$ have similar openings and are Pow $_{\mathrm{x}}$ equivalents to Pow $_{\mathrm{y}}$. When nodes $\mathrm{U}$ and $\mathrm{V}$ have pre-identified the possible RJ, they'll see if node points $\mathrm{X}$ and $\mathrm{Y}$ will adjust their transmission controls in accordance with values $\mathrm{D}_{\mathrm{x}}$ and $\mathrm{D}_{\mathrm{y}}$. Since only a single node has the capacity to modify the power that is transmitted, vehicle which will reassign the sending power is going to get picked out from it (vehicle in which transmitting slot opening and power is needed modification). The vehicles will be drawn out based on their ID values, and the collision will be compared to a typical result. If any one of the identification number is even among two, then one must quit and the vehicle point with the lower ID number will be selected in that case, as seen in Figure 1b, and it updates the transmission controls after tolerating the data note sent by V. As a consequence, an RJ is often cut off from deciding if the slot between $\mathrm{X}$ and $\mathrm{Y}$ is more unassuming than $2 \mathrm{Reg}_{1}$.

If the interval between nodes $\mathrm{X}$ and $\mathrm{Y}$ is less than $\operatorname{Reg}_{0}+\operatorname{Reg}_{1}$, an IRJ exists. Regardless, if there are neighbour node points that can know the potential influence, an IRJ is often used. Since both node points are one-hop neighbours of $\mathrm{V}$, node $\mathrm{V}$ analyses and finds out the information of the current state (e.g., location, running velocity, heading) among the vehicle $\mathrm{X}$ and $\mathrm{Y}$, as seen in Figure 1c. If distance $\operatorname{Reg}_{0}+\operatorname{Reg}_{1}$, where distance represents the space between $\mathrm{X}$ and $\mathrm{Y}$ after a brief cutoff (Threshold time) Tth, this should lead to the need for incorporating the current data of $X$ and $\mathrm{Y}$ vehicle, switching note will be given by V. One of the possible accident node points would be chosen by their ID numbers, much like the RJ cutoff. After receiving the slot swapping note, the selected node will reacquire another empty space.

Pre-identification of both RJ and IRJ necessitates the collaboration of neighbour node points, implying new increasing count of errors is often less; regardless, impacts do happen where there is no nodes satisfying node points are present. The evaluation of $\mathrm{D}_{\mathrm{x}}$ is critical for both RJ and IRJ precomputation, while the evaluation of Pow $_{x}$ is only needed for RJ pre-identification [1]. A one-bit flag is used to indicate whether the RJ and IRJ overhead demands more bandwidth or not. The welcoming node, which is a nearest neighbour among the proven vehicle point and anticipates propagation problems, can store notice data in the safety message, is it is not receiving any notice about a similar possible accident before its time opening occurs. If at least one note package is submitted, the chosen node point will play the update.

\section{Potential IRJ crash estimate}

The nearest vehicle of a particular node calculates IRJ among two other vehicles, which must analyze the identified IRJ should crash before notifying the slot switch. We request the possible IRJ collides with five instances, taking into account the locations and moving orientation of the nodes that can cause collision: two vehicles intersecting, one vehicle starting from the intersection and therefore leaving towards the meeting, two vehicles initiating the intermingling, another pair of nodes going with follow manner, and another pair of nodes going in the contrast direction. All five choices are considered, and simulations are run accordingly.

\section{SIMULATION, RESULT AND DISCUSSION}

The current segment displays with the findings from mat lab that is used for testing the appearance of our structures in highway and city scenarios where they vary from VeMac. Tth is estimated to be around $0.5 \mathrm{~s}$. Considering the channel state, the nodes that are nearer to the source node point should get packets that are available around the probability (0.98). Package transport rate, the Transmission number (Tx) crashes (typical amount in insufficient sending's per time slots; and the number of transmission (Tx) crashes (the typical number of insufficient transmissions per time frame; The sending up of the message is rendered useless when neighbours are unable to receive the community messages due to transmission collusion), and the number of receiving nodes (Rx) crashes (the Avg. number of unsuccessful packs per layout). The simulation parameters are described in Table 1.

We first look at the system's performance in a highway setting with varying cluster arrangements $(400,350,300$, and 250 nodes or vehicles in each areas). Each vehicle flies or moves by fixing a regular velocity, anywhere in-between 60 to 120 kilometers per hour. Nodes return to the path by already leaving the opposite path and then appear at the opposite side of the path, ensuring that nodes or vehicles that remains same. Sequenced timeslot openings on each configuration is 100 , and the figures for the number of packet collides 
Table 1. Simulation parameters

\begin{tabular}{|l|c|c|}
\hline \multicolumn{1}{|c|}{ Parameter } & Highway & City \\
\hline Road Length & $5000 \mathrm{~m}$ & $1030 \mathrm{~m}$ \\
\hline Number of Roads & 1 & $3+3$ \\
\hline $\begin{array}{l}\text { Number of Lanes } \\
\text { (each Direction) }\end{array}$ & 4 & 1 \\
\hline Lane Width & $5 \mathrm{~m}$ & $5 \mathrm{~m}$ \\
\hline Time & $0.5 \mathrm{~s}$ & $0.5 \mathrm{~s}$ \\
\hline Speed & $60-120 \mathrm{~km} / \mathrm{h}$ & $20-80 \mathrm{~km} / \mathrm{h}$ \\
\hline Communication range & $300 \mathrm{~m}$ & $300 \mathrm{~m}$ \\
\hline Frame length & $0.1 \mathrm{~s}$ & $0.1 \mathrm{~s}$ \\
\hline Simulation time & $3 \mathrm{~min}$ & $3 \mathrm{~min}$ \\
\hline
\end{tabular}

are seen in Figure 2. When compared to VeMac, our system performs better, with 32 percent, 25 percent, 16 percent fewer Tx data collisions and 63 percent, 46 percent, 27 percent fewer Rx data collisions, exclusively for $\operatorname{Reg}_{0}$ assessments of $0.25 \mathrm{Reg}_{1}, 0.5 \mathrm{Reg}_{1}$, and $0.75 \mathrm{Reg}_{1}$ during when the total vehicles in this area is 400 .

When signaling issues are lowered the power P0 will be considered, so the collision rate is decreased. Meanwhile, investment among the nodes also pre-identifies and removes such possible consolidating results. In comparison regarding the packets losses in $\mathrm{Tx}$, corresponding $\mathrm{Reg}_{\mathrm{x}}$ sway amount is slightly lower. This is also due to the fact that by using power $\mathrm{P}_{0}$, the propagation range is reduced, and the packs aren't received by the farthest nodes. As a result, the $\mathrm{R}_{0}$ evaluation should be rendered with caution, as shown by the criteria of safety-related applications. The effects of the bundle movement rate are seen in Figure 3. When the thickness is high, our arrangement produces a superior presentation. For 400 vehicles for each bearing, the transport volume increases by $11 \%, 8 \%$, and $5 \%$, respectively.

At that time, we have looked at the impact by numbered spaces per current frame in this design. Amount of nodes operating in town is considered to be 250 for each length, with 60 , $70,80,90$, and 100 spaces, as in Figure 1. The findings as seen in Figure 4. When the number of openings as in Figure 1 is 60, we can see that the number of accidents for each arrangement is high. This happens often since the amount of available spaces is inadequate. Regardless, as compared to the VeMac, our setup has 27 percent, 21 percent, and 12 percent fewer Tx impacts and 64 percent, 46 percent, 25 percent lesser collisions in the receiver side messages. When the amount of time slot opening reaches 100 , then gradually throughput decreases. When seen in comparison to VeMac, our arrangement actually has a lower collision rate.

The package movement rate for various layouts varying with numbers of openings. We will see that our setup outperforms VeMac, particularly when the number of openings per layout is minimal. As the number of spaces per configuration is set to 60 , an unprecedented for the first

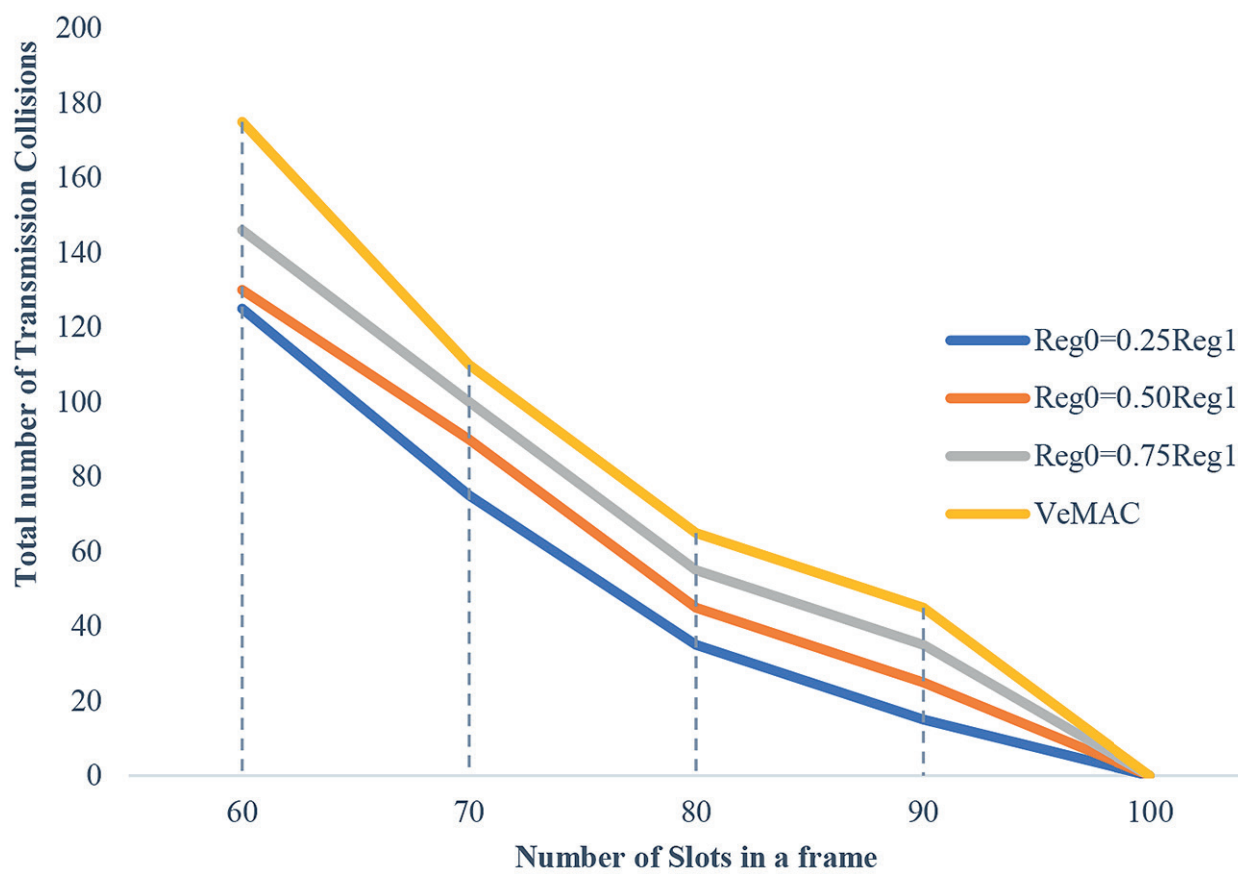

Fig. 2. The total number of slots and its collisions 


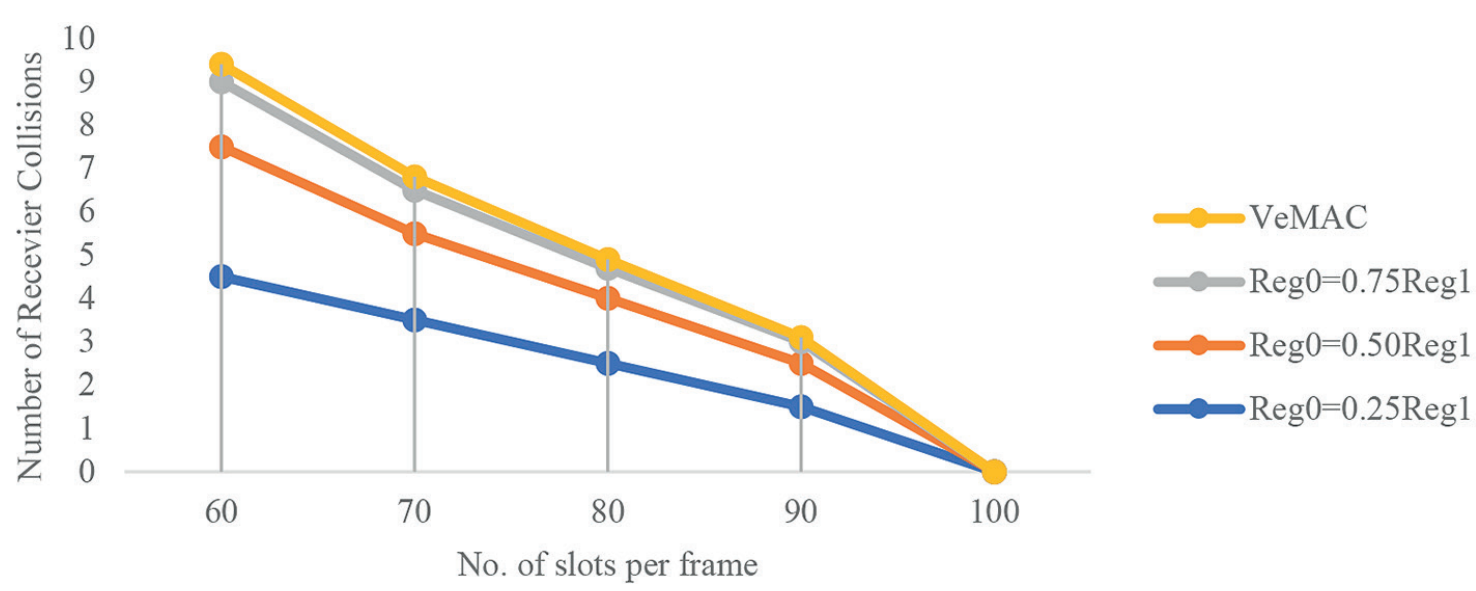

Fig. 3. The total number of slots and its receiver collisions

component execution of 13 percent, 9 percent, and 5 percent higher transport rate than VeMac is often obtained when the $\mathrm{Reg}_{0}$ assessments are set to $0.25 \mathrm{Reg}_{1}, 0.5 \mathrm{Reg}_{1}$, and $0.75 \mathrm{Reg}_{1}$, respectively.

The city condition may be a grid system has both at vertical and horizontal places. Every node flies at a regular velocity, anywhere in-between 20 to 80 kilometers per hour. The number of time slots for a node will range between 60 to $100 \mathrm{oc}-$ cupancy. Compare to results obtained in [12], our result give better performance. When the assessments of $\mathrm{R} 0$ are set to $0.25 \mathrm{R} 1,0.5 \mathrm{R} 1$, and $0.75 \mathrm{R} 1$ with a thickness of 500 cars, we can find that our arrangement has better execution when compared to VeMac, with 41 percent, 33 percent, and 25 percent less Tx impacts and 63 percent, 49 percent, and 32 percent less Rx collisions, respectively. The transport rate increases by $10 \%, 8 \%$, and $4 \%$, respectively, when the various $\operatorname{Reg}_{0}$ assessments are taken into account.

\section{CONCLUSION}

By separating observational areas, we proposed a completely new approach for improving propagation performance with simple amount of VANET BSM's. Our scheme increased the state of consciousness to the nearby nodes while accepting small deterioration to distant nodes, responding for realistic environments. In comparison to standard MAC protocols, nodes will be using the same slots in the nearby area around the RSU by compromising the consciousness quality of remote vehicles. As a result, in high-density networks, our scheme worked well. Meanwhile, possible merging collisions may be identified in advance and prevented by node cooperation. Our techniques greatly enhanced the network's efficiency, as shown by simulation results.

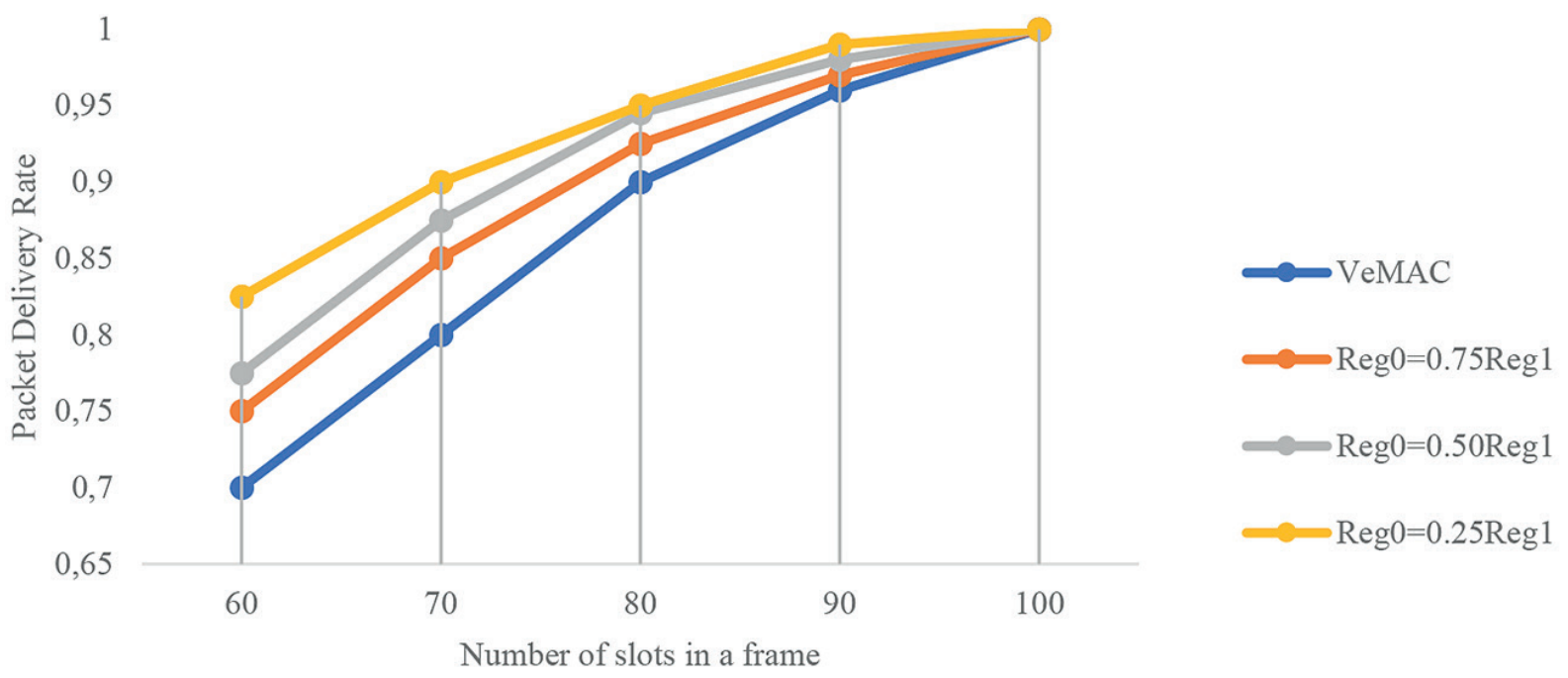

Fig. 4. The total number of slots vs packet delivery rate 


\section{Acknowledgements}

This work was sponsored by Indian Council of Medical Research - ICMR (Ref: Adhoc/113/2017/HSR).

\section{REFERENCES}

1. Alotaibi M.M., Mouftah H.T. Relay Selection for Heterogeneous Transmission Powers in VANETs, IEEE. Access. 2017; 5: 4870-4886. DOI: 10.1109/ ACCESS.2017.2679606

2. Cao S., Lee V.C.S. A Novel Adaptive TDMABased MAC Protocol for VANETs, IEEE Commun. Lett. 2018; 22(3), 614-617. DOI: 10.1109/ LCOMM.2017.2785378

3. Chen Y.D., Shih Y.P., Shih K.P. An emergency message dissemination protocol using N-way search with power control for VANETs, IEEE International Conference on Communications (ICC), London 2015, 3653-3658. DOI: 10.1109/ ICC.2015.7248892

4. Dressler F., Klingler F., Sommer C., Cohen R. Not All VANET Broadcasts Are the Same: ContextAware Class Based Broadcast, IEEE/ACM Trans. Networking. 2018; 26(1): 17-30. DOI: 10.1109/ TNET.2017.2763185

5. Hadded M., Muhlethaler P., Laouiti A., Zagrouba R., Saidane L.A. TDMA-Based MAC Protocols for Vehicular Ad Hoc Networks: A Survey, Qualitative Analysis, and Open Research Issues, IEEE Commun. Surv. Tutorials. 2015; 17(4), 2461-2492. DOI: 10.1109/COMST.2015.2440374

6. Nguyen V., Oo T.Z., Chuan P., Hong C.S. An Efficient Time Slot Acquisition on the Hybrid TDMA/CSMA Multichannel MAC in VANETs, IEEE Commun. Lett. 2016; 20(5): 970-973. DOI:

\subsection{9/LCOMM.2016.2536672}

7. Peng F., Zhang G., Huang X., Ye X., Wu M. A Novel TDMA-MAC Protocol for VANET Using Cooperative and Opportunistic Transmissions, IEEE 82nd Vehicular Technology Conference (VTC2015Fall), Boston, MA, USA 2016, 1-2. DOI: 10.1109/ VTCFall.2015.7390806

8. Rawat D.B., Popescu D.C., Yan G., Olariu S. Enhancing VANET Performance by Joint Adaptation of Transmission Power and Contention Window Size. IEEE Trans. Parallel Distrib. Syst. 2011; 22(9): 1528-1535. DOI: 10.1109/TPDS.2011.41

9. Rezgui J., Cherkaoui S. Analytical transmit power adjustment in cooperative vehicle safety systems, IEEE Global Communications Conference (GLOBECOM), Anaheim, CA, USA 2012, 597-602. DOI: 10.1109/GLOCOM.2012.6503178

10. Tahmasbi-Sarvestani A., Fallah Y.P., Kulathumani V. Network-Aware Double-Layer Distance-Dependent Broadcast Protocol for VANETs, IEEE Trans. Veh. Technol. 2015; 64(12), 5536-5546. DOI: 10.1109/TVT.2015.2487998

11. Zemouri S., Djahel S., Murphy J. Smart adaptation of beacons transmission rate and power for enhanced vehicular awareness in VANETs, 17th International IEEE Conference on Intelligent Transportation Systems (ITSC), Qingdao, China 2014, 739-746. DOI: 10.1109/ITSC.2014.6957777

12. Zhang X.M., Yan L., Zhang H., Sung D.K. A Concurrent Transmission Based Broadcast Scheme for Urban VANETs, IEEE Trans. on Mobile Comput. 2019; 18(1), 1-12, DOI: 10.1109/TMC.2018.2827930 